\title{
Sur une inégalité différentielle à l'argument retardé
}

\author{
par K. Zima (Katowice)
}

Soit l'équation différentielle de la forme

$$
y^{\prime}(t)=\int_{0}^{\infty} F(y(t-s)) d r(t, s)+f(t), \quad t \epsilon[A, B), \quad B \leqslant+\infty .
$$

Pour $t \leqslant A$ on a $y(t)=\varphi(t)([2]$, p. 53).

Sur l'équation (1) nous faisons les hypothèses suivantes:

(a) $F(z)$ est une fonction définie et nondécroissante pour $z \in(-\infty,+\infty)$.

(b) $r(t, s)$ est une fonction définie dans l'ensemble $0 \leqslant s<\infty$, $A \leqslant t<B$ et nondécroissante par rapport à $s$ pour chaque $t \in[A, B)$.

(c) $f(t)$ est une fonction définie dans l'intervalle $[A, B)$.

Moyennant ces hypothèses nous allons démontrer le théorème suivant:

THÉoRغ̀me I. Si la fonction $z(t)$ est différentiable et satisfait aux conditions suivantes

$$
\begin{gathered}
z(t) \geqslant \varphi(t) \quad \text { pour } \quad t \leqslant A \\
z^{\prime}(t)>\int_{0}^{\infty} F(z(t-s)) d r(t, s)+f(t) \quad \text { pour } \quad t \in[A, B),
\end{gathered}
$$

alors $y(t)<z(t)$ pour $t \epsilon(A, B)$, où $y(t)$ est une solution de l'équation (1) avec la fonction initiale $\varphi(t)$.

Démonstration. Des hypothèses $(\alpha),(\mathrm{a}),(\mathrm{b}),(\beta)$ résulte l'inégalité

$$
\begin{aligned}
z^{\prime}(A) & >\int_{0}^{\infty} F(z(A-s)) d r(A, s)+f(A) \\
& \geqslant \int_{0}^{\infty} F(\varphi(A-s)) d r(A, s)+f(A)=y^{\prime}(A) .
\end{aligned}
$$

Nous avons done

$$
\begin{gathered}
z^{\prime}(A)>y^{\prime}(A), \\
z(A) \geqslant \varphi(A)=y(A) .
\end{gathered}
$$


De l'inégalité (i) et (ii) résulte l'existence d'un intervalle $(A, \tau)$ tel que $z(t)>y(t)$ si $t \in(A, \tau)$. Soit $\tau_{0}$ la plus grande des valeurs de $t$ pour lesquelles l'inégalité $z(t)>y(t)$ a lieu, si $t \epsilon\left(A, \tau_{0}\right)$. Pour le point $\tau_{0}$ nous obtenons les relations

$$
\begin{gathered}
z\left(\tau_{0}\right)=y\left(\tau_{0}\right), \\
z^{\prime}\left(\tau_{0}\right) \leqslant y^{\prime}\left(\tau_{0}\right) .
\end{gathered}
$$

D'autre part, en vertu de l'hypothèse $(\beta)$ nous obtenons

$$
\begin{aligned}
z^{\prime}\left(\tau_{0}\right) & >\int_{0}^{\infty} F\left(z\left(\tau_{0}-s\right)\right) d r\left(\tau_{0}, s\right)+f\left(\tau_{0}\right) \\
& \geqslant \int_{0}^{\infty} F\left(y\left(\tau_{0}-s\right)\right) d r\left(\tau_{0}, s\right)+f\left(\tau_{0}\right)=y^{\prime}\left(\tau_{0}\right) .
\end{aligned}
$$

Ainsi $z^{\prime}\left(\tau_{0}\right)>y^{\prime}\left(\tau_{0}\right)$, en contradiction avec l'inégalité $(\mathrm{jj})$. Point $\tau_{0}$ ne peut donc pas appartenir à l'intervalle $(A, B)$, ce qui démontre le théorème $\mathrm{I}$.

THÉoRغ̀me II. Si dans le théorìme I nous remplaçons les hypothèses $(\alpha),(\beta)$ par les suivantes

$$
\begin{gathered}
z(t) \leqslant \varphi(t) \quad \text { pour } t \leqslant A, \\
z^{\prime}(t)<\int_{0}^{\infty} F(z(t-s)) d r(t, s)+f(t) \quad \text { pour } \quad t \in[A, B),
\end{gathered}
$$

on peut démontrer d'une manière analogue que dans l'intervalle $(A, B)$ on a l'inégalité $z(t)<y(t)$.

Application des théorèmes I et II à l'évaluation des intégrales des equations correspondantes. Nous admettons pour l'équation (1) les mêmes hypothèses (a), (b), (c) que dans les théorèmes I et II et de plus, nous admettons

(d) $\int_{0}^{\infty} d r(t, s)$ est convergente pour chaque $t \in[A, B)$,

(e) $\sup \varphi(t)=K<+\infty$.

Comparons à l'équation (1) l'équation différentielle sans argument retardé

$$
z^{\prime}=|F(z)| \cdot M(t)+|f(t)|+\varepsilon,
$$

où $M(t)=\int_{0}^{\infty} d r(t, s), \varepsilon>0$ arbitraire. En nous appuyant sur ces hypothèses nous allons démontrer le théorème suivante:

THÉoRغ̀me III. Si $z(t)$ est pour $t \in[A, B)$ une intégrale de l'équation (2) avec la condition initiale $z(A) \geqslant K$ et $z(t)=z(A)$ quand $t \leqslant A$, et si, de plus, $y(t)$ est une intégrale de l'équation (1) dans l'intervalle $[A, B)$ pour la fonction initiale $\varphi(t)$, alors pour $t \in(A, B)$ on a l'inégalité $y(t)<z(t)$. 
Démonstration. Il résulte de la définition de la fonction $M(t)$ et de l'hypothèse (b) que $M(t) \geqslant 0$. Done chaque intégrale de l'équation (2) est une fonction nondécroissante. En vertu de ce fait et des hypothèses (a), (b) nous obtenons

$$
\begin{aligned}
\int_{0}^{\infty} F(z(t-s)) d r(t, s)+f(t) & \leqslant \int_{0}^{\infty} F(z(t)) d r(t, s)+f(t) \\
& <|F(z(t))| \cdot M(t)+f(t)+\varepsilon=z^{\prime}(t) .
\end{aligned}
$$

La fonction $z(t)$ satisfait donc à l'inégalité $(\alpha)$ et $(\beta)$. Les hypothèses $\mathrm{du}$ théorème $\mathrm{I}$ sont donc vérifiées; pour $t \in(A, B)$ on a done l'inégalité $y(t)<z(t)$, ce qu'il fallait démontrer.

THÉORغ̀me IV. Si dans le théorème III on remplaçe l'hypothèse (e) par l'hypothèse $(\mathrm{e}) \inf \varphi(t)=k$, alors dans l'intervalle $(A, B)$ on a l'inégalité $z(t)<y(t)$, où $z(t)$ est une intégrale de l'équation

$$
\begin{aligned}
z^{\prime}=-|F(z)| \cdot M(t)-|f(t)|-\varepsilon, \quad z(A) \leqslant k \\
\text { et } \quad z(t)=z(t) \quad \text { pour } \quad t \leqslant A,
\end{aligned}
$$

et $y(t)$ est une intégrale de l'équation (1) avec la fonction initiale $\varphi(t)$.

Exemple. Si l'on pose $F^{\prime}(z) \equiv z$, l'équation (1) prendra la forme

$$
y^{\prime}(t)=\int_{0}^{\infty} y(t-s) d r(t, s)+f(A)
$$

Si, de plus, la fonction initiale $\varphi(t)$ satisfait à la condition $\sup |\varphi(t)|=K>0$ alors, en vertu des théorèmes III et IV, pour l'intégrale $y(t)$ de l'équation $\left(1^{*}\right)$ nous aurons l'évaluation

$$
z_{1}(t)<y(t)<z_{2}(t) \quad \text { pour } \quad t \in(A, B),
$$

où $z_{1}(t)$ est une intégrale de l'équation $z^{\prime}=M(t) \cdot z-|f(t)|-\varepsilon$ avec la condition initiale $z_{1}(A)=-K$, et $z_{2}(t)$ est une intégrale de l'équation $z^{\prime}=M(t) z$ $+f(t)+\varepsilon$, avec la condition $z_{2}(A)=K$.

Après avoir effectivement déterminé la fonction $z_{1}(t)$ et $z_{2}(t)$, et passé à la limite avec $\varepsilon$, nous obtenons l'inégalité suivante

$$
|y(t)| \leqslant \exp \left[\int_{\boldsymbol{A}}^{t} M(s) d s\right] \cdot\left\{\int_{\boldsymbol{A}}^{t}|f(s)| \cdot \exp \left[-\int_{\mathcal{A}}^{s} M(\tau) d \tau\right] d s+K\right\}
$$

L'évaluation donnée ici été démontrée d'une autre manière par A. D. Myszkis ([2], page 55). 
Généralisation des théorèmes I-IV aux systèmes d'inégalités différentielles. Considérons le système d'équations différentielles de la forme

$$
\begin{aligned}
& y_{v}^{\prime}(t)=\int_{0}^{\infty} F_{v}\left(y_{1}(t-s), \ldots, y_{n}(t-s)\right) d r_{v}(t, s)+f_{v}(t), \quad t \in[A, B), \\
& y_{v}(t)=\varphi_{v}(t) \quad \text { pour } \quad t \leqslant A, \quad v=1,2, \ldots, n .
\end{aligned}
$$

Pour le système $\left(1^{\circ}\right)$ nous admettons les hypothèses suivantes

$\left(\mathrm{a}^{0}\right) F_{v}\left(u_{1}, \ldots, u_{n}\right)$ est une fonction définie pour $-\infty<u_{p}<+\infty$,

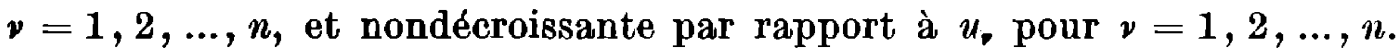

$\left(\mathrm{b}^{\circ}\right) r_{v}(t, s)$ est une fonction définie pour $s \in[0,+\infty)$ et $t \in[A, B)$ et nondécroissante par rapport à $s$ pour $t \in[A, B)$.

$\left(c^{0}\right) f_{v}(t)$ est une fonction définie pour $t \in[A, B), v=1,2, \ldots, n$.

Moyennant ces hypothèses nous allons démontrer le théorème suivant:

THÉOR亡̀me $\mathrm{I}^{\mathrm{0}}$. Soient $z_{\nu}(t), v=1,2, \ldots, n$, des fonctions définies dans l'intervalle $(-\infty, B)$ differentiables dans l'intervalle $[A, B)$ et satisfaisant aux conditions suivantes

$$
\begin{gathered}
z_{v}(t) \geqslant \varphi_{v}(t) \quad \text { pour } \quad t \leqslant A, \quad v=1,2, \ldots, n, \\
z_{\nu}^{\prime}(t)>\int_{0}^{\infty} F_{\nu}\left(z_{1}(t-s), \ldots, z_{n}(t-s)\right) d r_{v}(t, s)+f_{\nu}(t), \quad t \in[A, B), \\
v=1,2, \ldots, n .
\end{gathered}
$$

Alors dans l'intervalle $(A, B)$ nous avons les inégalités $z_{v}(t)>y_{\bullet}(t)$, $\nu=1,2, \ldots, n$, où $\left\{y_{1}(t), y_{2}(t), \ldots, y_{n}(t)\right\}$ est une intégrale du système $\left(1^{\circ}\right)$ avec les functions initiales $\varphi_{1}(t), \varphi_{2}(t), \ldots, \varphi_{n}(t)$.

Démonstration. Des hypothèses $\left(a^{\circ}\right),\left(b^{\circ}\right),\left(\alpha^{0}\right)$ résulte que pour chaque $\nu=1,2, \ldots, n$, on a

$$
z_{\nu}^{\prime}(A)>\int_{0}^{\infty} F_{\nu}\left(\varphi_{1}(A-s), \ldots, \varphi_{n}(A-s)\right) d r_{\nu}(A, s)+f_{v}(A)=y_{\nu}^{\prime}(A), \quad \text { c.à.d. , }
$$

$$
\begin{array}{cc}
z_{v}^{\prime}(A)>y_{v}^{\prime}(A) \quad \text { pour } & v=1,2, \ldots, n, \\
z_{v}(A) \geqslant \varphi_{\nu}(A)=y_{v}(A), & v=1,2, \ldots, n .
\end{array}
$$

Il résulte de l'inégalité $\left(\mathrm{i}^{\circ}\right)$ et (ii $\left.{ }^{\circ}\right)$ qu'il existe des nombres $\tau_{v}, v=1,2, \ldots, n$, tels que pour chaque $v$ on a l'inégalité $z_{v}(t)>y_{v}(t)$ lorsque $t \epsilon\left(A, \tau_{v}\right)$.

Soit $\tau_{\nu}^{0}$ la borne supérieure des valeurs de $t$, pour lesquelles l'inégalité $z_{v}(t)>y_{v}(t)$ est verifiée, si $t \in\left(A, \tau_{p}^{0}\right)$. Soit de plus $\tau_{\sigma}^{0}=\min \left(\tau_{1}^{0}, \tau_{2}^{0}, \ldots, \tau_{n}^{0}\right)$. Nous allons montrer que $\tau_{\sigma}^{0}$ n'appartient pas à l'intervalle $(A, B)$. Il est évident que le point $\tau_{\sigma}^{0}$ satisfait aux relations

$$
\begin{aligned}
& z_{\sigma}\left(\tau_{\sigma}^{0}\right)=y_{\sigma}\left(\tau_{\sigma}^{0}\right), \\
& z_{\sigma}^{\prime}\left(\tau_{\sigma}^{0}\right) \leqslant y_{\sigma}^{\prime}\left(\tau_{\sigma}^{0}\right) .
\end{aligned}
$$


D'autre part, en vertu de l'hypothèse $\left(\beta^{\circ}\right)$ nous obtenons

$$
\begin{aligned}
z_{\sigma}^{\prime}\left(\tau_{\sigma}^{0}\right) & >\int_{0}^{\infty} F_{\sigma}\left(z_{1}\left(\tau_{\sigma}^{0}-s\right), \ldots, z_{n}\left(\tau_{\sigma}^{0}-s\right)\right) d r_{\sigma}\left(\tau_{\sigma}^{0}, s\right)+f_{\sigma}\left(\tau_{\sigma}^{0}\right) \\
& \geqslant \int_{0}^{\infty} F_{\sigma}\left(y_{1}\left(\tau_{\sigma}^{0}-s\right), \ldots, y_{n}\left(\tau_{\sigma}^{0}-s\right)\right) d r\left(\tau_{\sigma}^{0}, s\right)+f_{\sigma}\left(\tau_{\sigma}^{0}\right)=y_{\sigma}^{\prime}\left(\tau_{\sigma}^{0}\right),
\end{aligned}
$$

c.à.d.

$$
z_{\sigma}^{\prime}\left(\tau_{\sigma}^{0}\right)>y_{\sigma}^{\prime}\left(\tau_{\sigma}^{0}\right)
$$

L'inégalité obtenue est en contradiction avec l'inégalité $\left(\mathrm{jj}^{\mathrm{O}}\right)$ se qui prouve que le point $\tau_{\sigma}^{0}$ n'appartient pas à l'intervalle $(A, B)$.

Donc, dans tout l'intervalle $(A, B)$ on a l'inégalité $z_{v}(t)>y_{v}(t)$, $v=1,2, \ldots, n$.

On démontre d'une manière analogue le théorème suivant:

THÉORÈME II ${ }^{\circ}$. Nous admettons sans modifications les hypothèses $\left(\mathrm{a}^{0}\right)$, $\left(\mathrm{b}^{0}\right),\left(\mathrm{c}^{\circ}\right)$ du théorème $\mathrm{I}^{\circ}$ et dans les hypothèses $\left(\alpha^{\circ}\right)$ et $\left(\beta^{\circ}\right)$ nous changeons dans le sens des inégalités, e'est-à-dire

$$
\begin{gathered}
z_{v}(t) \leqslant \varphi_{v}(t), \quad t \leqslant A, \quad v=1,2, \ldots, n \\
z_{v}^{\prime}(t)<\int_{0}^{\infty} F_{\nu}\left(z_{1}(t-s), \ldots, z_{n}(t-s)\right) d r_{v}(t, s)+f_{\nu}(t), \quad t \in[A, B) .
\end{gathered}
$$

Alors on a pour $t \epsilon(A, B)$ l'inégalité $z_{\nu}(t)<y_{\nu}(t), v=1,2, \ldots, n$.

Evaluation des intégrales du système $\left(1^{\circ}\right)$ par une intégrale du système correspondant d'équations différentielles ordinaires. On peut associer univoquement au système $\left(1^{\circ}\right)$ le système suivant sans argument retardé

$$
u_{\nu}^{\prime}= \pm\left\{\left|F_{v}\left(u_{1}, \ldots, u_{n}\right)\right| \cdot M_{\nu}(t)+\left|f_{v}(t)\right|+\varepsilon_{v}\right\}, \quad v=1,2, \ldots, n
$$

où $M_{v}(t)=\int_{0}^{\infty} d r_{v}(t, s)$ et $\varepsilon_{v}>0$ est d'ailleurs arbitraire. Nous allons formuler le theoreme suivant:

THÉonème $\operatorname{III}^{\circ}\left(\mathrm{IV}^{\circ}\right)$. S'il existe dans l'intervalle $[A, B)$ une solution $\left\{u_{1}(t), \ldots, u_{n}(t)\right\}$ du système $\left(2^{\circ}\right)$ avec la condition initiale $u_{\nu}(A) \geqslant \sup \varphi_{v}(t)$ $\left(u_{v}(A) \leqslant \inf \varphi_{v}(t)\right)$ pour $v=1,2, \ldots, n$, alors dans l'intervalle $(A, B)$ on. a l'inégalité

$$
u_{v}(t)>y_{v}(t) \quad\left(u_{v}(t)<y_{v}(t)\right) \quad v=1,2, \ldots, n,
$$


où $\left\{y_{1}(t), y_{2}(t), \ldots, y_{n}(t)\right\}$ est une solution du système $\left(1^{\circ}\right)$ avec les fonctions initiales $\varphi_{1}(t), \ldots, \varphi_{n}(t)$.

Le démonstration du théorème $\operatorname{III}^{\circ}\left(\mathrm{IV}^{\circ}\right)$ est analogue à celle du théorème III (IV).

\section{Travaux cités}

[1] E. Kamke, Differentialgleichungen reeller Funktionen, Leipzig 1930.

[2] А. Д. Мышкис, Линейные дифференииалные уравнения с запаздываюичи аргументом, Моцква-Ленинград 1951.

Reçu par la Rédaction le 1.3.1961 